\title{
Eosinophilic pleural effusion: A case and a review
}

\author{
Mark D Lacy MD, FIDSA
}

\begin{abstract}
A previously healthy middle-aged woman presented with evanescent skin lesions and bilateral pleural effusions with an eosinophilic predominance. Following this case summary, a description of eosinophilic pleuritis, the epidemiology, etiologic considerations, and selected therapies for this syndrome are discussed. Eosinophilic pleural effusion is caused by myriad etiologies and is a therapeutic challenge.
\end{abstract}

Keywords: pleural effusion, eosinophilic effusion, dermatitis

\section{INTRODUCTION}

Pleural effusions are a common clinical problem in adult medicine, but an effusion with a cellular component comprised of many eosinophils is not. Eosinophilic pleural effusions (EPE) have been attributed to a wide variety of etiologic agents, including infections, adverse drug reactions, malignancy, and chest trauma. Even after investigating these causes, the cause of an EPE can still be uncertain. After presentation of a case, an overview of the causes of EPE is discussed followed by comments on therapy.

\section{CASE}

A 37-year-old Hispanic homemaker developed left sided pleuritic chest pain over a few days in the fall. This was associated with malaise, fatigue, and some sweats but no fever or chills. She was admitted to a hospital and diagnosed with pneumonia for which she received quinolone and macrolide antibiotics. She didn't respond, and dyspnea developed. Echocardiography was normal. She was transferred

Corresponding author: Mark Lacy Contact Information: Mark.lacy@ttuhsc.edu DOI: 10.12746/swrccc.v8i33.633 to University Medical Center in Lubbock, Texas, when computed tomography of the thorax showed pleural effusions. She had no cough or other focal symptoms except for evanescent skin lesions, occurring over the prior weeks, primarily on the trunk and proximal extremities. These were not pruritic or triggered by trauma or sun exposure. She had no past medical history, medication use, or social history relevant to this presentation. She had traveled to California several months prior to presentation.

She had BMI of $33.6 \mathrm{~kg} / \mathrm{m}^{2}$, a normal temperature, and normal vital signs except for a respiratory rate of 20 breaths per minute with $91 \%$ oxygen saturation on room air. Reduced breath sounds were present at both bases. There were several scattered annular erythematous plaques on the abdomen and proximal thighs (Figure 1). The remainder of her examination was within normal limits showed no adenopathy.

Her leukocyte count was $6,170 / \mu \mathrm{L}$ ( $75 \%$ neutrophils, $20 \%$ lymphocytes, $4 \%$ monocytes, $\%$ eosinophils), hemoglobin was $9.4 \mathrm{gm} / \mathrm{dL}$ (MCV $76 \mathrm{fL}$ ), and the platelet count was normal. C-reactive protein was $12.1 \mathrm{mg} / \mathrm{dL}$. Serum chemistry profile was normal. Both the chest radiograph (Figure 2) and chest computed tomography (Figure 3) showed bilateral pleural effusions and possible lower lung consolidations. Transthoracic echocardiography showed a small pericardial effusion but no other abnormalities. Thoracentesis recovered pleural 


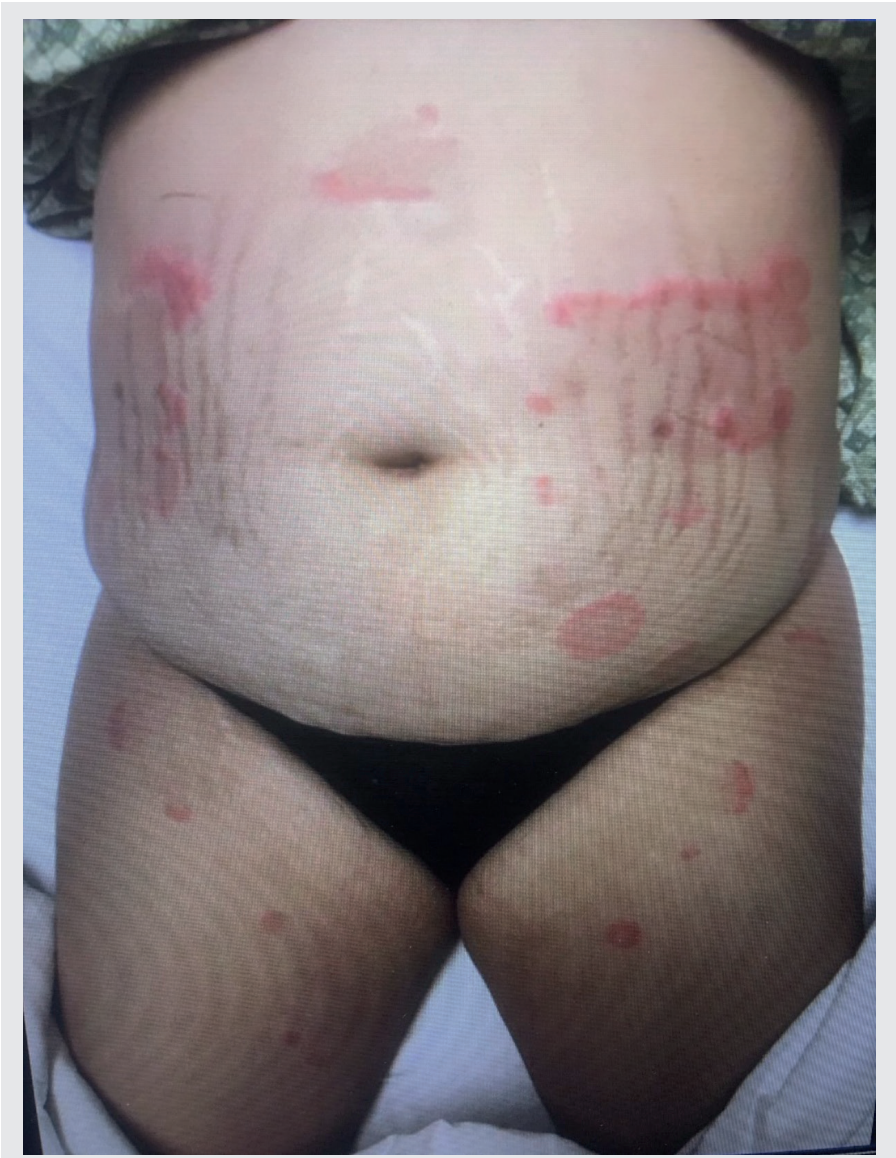

Figure 1. Annular erythematous plaques on the abdomen and thighs.

fluid with 3200 red cells $/ \mathrm{mm}^{3}$ and 2558 leukocytes $/ \mathrm{mm}^{3}$ (84\% eosinophils, $9 \%$ lymphocytes, $7 \%$ monocytes). Pleural fluid LDH was $663 \mathrm{IU} / \mathrm{L}$ (corresponding serum value: $245 \mathrm{IU} / \mathrm{L}$ ), and pleural fluid protein was $4.9 \mathrm{~g} / \mathrm{dL}$. Gram stain was negative, and cultures for bacterial and fungi had no growth. Investigations conducted over the subsequent week for infectious, autoimmune, and neoplastic conditions are summarized in Table 1. The skin lesions lasted a few days, and then similar lesions appeared at various other sites over the next week.

During this hospitalization, the effusions increased in size. She was started on prednisone with rapid reduction in symptoms and no further increase in the pleural fluid. Her skin lesions completely disappeared. Following hospitalization, the prednisone

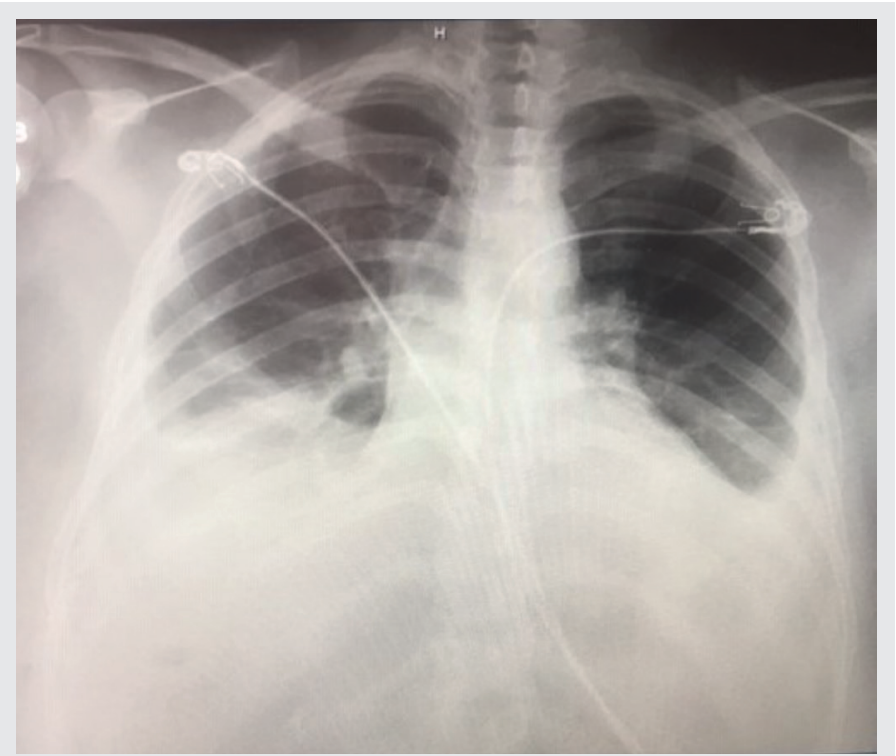

Figure 2. Portable A-P view of the chest reveals bilateral pleural effusions.

was tapered over several weeks, but at a lower dose, symptoms and effusions returned. This occurred over the next month at which point she had right videoassisted thoracoscopy which showed "murky fluid" but normal lung adherent to the pleura. Pleural biopsy and mechanical pleurodesis followed without complications. Pathology showed fibrinoid eosinophilic inflammatory infiltrates in the pleura. Lung tissue had patchy alveolar wall fibrosis at subpleural areas, but no eosinophilic alveolar process was apparent beyond the pleural-lung junction. No granulomas, vasculitic features, or malignant cells were seen (Figure 4).

High dose prednisone therapy reduced her symptoms, including the rash, and reduced her pleural effusions. However, whenever the corticosteroid dose was reduced, her conditioned worsened. Two months after the start of her therapy she developed a pericardial effusion, without eosinophilia in the pericardial fluid, which resolved with a pericardial window. She remained without cough, fever, arthralgias, or lymphadenopathy. Ultimately immunosuppression with steroid-sparing regimen consisting of colchicine and hydroxyurea was required to control her disease. Two years later she has fatigue but is asymptomatic with no recurrent effusion or skin lesions. 


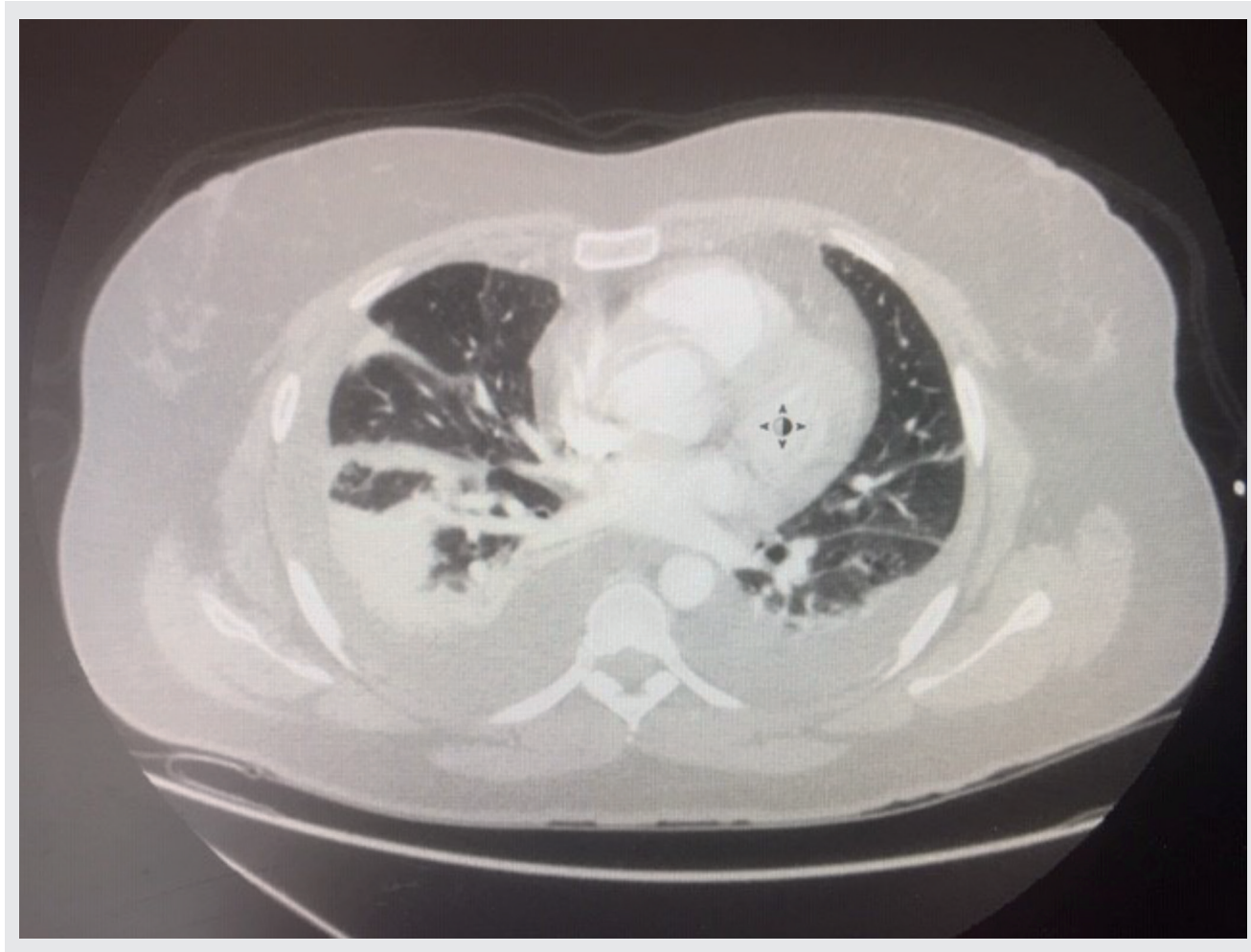

Figure 3. Computed tomography of the thorax reveals bilateral pleural effusions and an infiltrate at the right base.

\section{Discussion}

Eosinophilic pleural effusions can present both diagnostic challenges and difficult therapeutic decisions. The eosinophil is activated by $\mathrm{T}$ lymphocytes and mast cells, particularly upon release of interleukin (IL)-3 and IL-5; the latter prolongs the survival of eosinophils. Eosinophilia exists when $3 \%$ or more of peripheral blood leukocytes are eosinophils or when the absolute number is over 350 cells per cubic millimeter. An eosinophilic pleural effusion (EPE) is present when over $10 \%$ of the nucleated cells are eosinophils. ${ }^{1}$ Eosinophilic pleural effusion results with increased marrow production of eosinophils which migrate into and persist in the pleura. The migration and residence in a tissue is related to expression of vascular cell adhesion molecule-1. ${ }^{1}$

Eosinophilic pleural effusions are a relatively uncommon problem. In a retrospective review of over 2,200 European patients with pleural effusions, from 1995 to 2007 , only $7.2 \%$ had EPE. ${ }^{1}$ The etiology of pleural effusions is unknown in $10-20 \%$ of cases. With EPE the proportion of cases due to an unknown etiology may be even higher. In most studies in which an etiology is identified for EPE, malignancies are ranked near the top with lung and breast cancers among the most common followed by lymphomas and a variety of other solid and gastrointestinal neoplasms. An EPE has developed in a patient after mediastinal radiation therapy for Hodgkin's disease. The degree of eosinophilia in the pleural fluid tends to be less in the neoplastic causes than other etiologies. ${ }^{1} \mathrm{~A}$ summary of some of the common causes of EPE is shown in Table 2.

Infectious disease etiologies for EPE typically include parapneumonic effusions from pneumonia and Mycobacterium tuberculosis (MTB). The proportion of pleural effusions caused by MTB reflects the prevalence of tuberculosis in the patient population. In Tunisia, where tuberculosis is prevalent and pleural TB relatively common, nearly $40 \%$ of pleural effusions were attributed to MTB, whereas a series of 
Table 1. Diagnostic tests in the patient in this case report

\begin{tabular}{|l|l|}
\hline Study & Result/Interpretation \\
\hline ANA, c-ANCA antibody & Negative \\
\hline $\begin{array}{l}\text { Antimyeloperoxidase, Antiproteinase } 3 \\
\text { antibody }\end{array}$ & Negative \\
\hline Strongyloides antibody & Negative \\
\hline $\begin{array}{l}\text { Interferon gamma-release assay M. } \\
\text { tuberculosis }\end{array}$ & Negative \\
\hline HIV antibody & Negative \\
\hline Syphilis screen & Negative \\
\hline HCV antibody, HBs antigen & Negative \\
\hline Coccidioidiomycosis serology & Negative \\
\hline Histoplasma urine antigen & Negative \\
\hline Beta D glucan antigen (serum) & Negative \\
\hline Procalcitonin & 0.3 ng/ml \\
\hline Urine drug screen & Negative \\
\hline Bone marrow biopsy & Red cell hypochromia, 1\% eosinophils; normal \\
\hline Skin biopsy & Lichenoid dermatitis with eosinophils \\
\hline C3, C4 & Normal \\
\hline CH50 & $>60$ (elevated) \\
\hline JAK2 & Negative \\
\hline CT chest angiography & Normal \\
\hline Interleukin 5 & $<5$ pg/ml \\
\hline FISH, HES/4q12 & Negative for FIP1L1- PDGFRA rearrangement \\
\hline
\end{tabular}

patients in Poland included just over $2 \%$ caused by MTB. ${ }^{1,4}$ Pleural effusions associated with pulmonary tuberculosis or pleuritis typically have a lymphocytic predominance with $<10 \%$ eosinophils. If the proportion is greater, tuberculous effusion is unlikely. In patients with "Valley Fever," caused by the mycosis Coccidioides immitis or C. posadasii endemic to the southwestern deserts of the United States, northern Mexico, and portions of Central and South America, a mild peripheral eosinophilia may occur in $25 \%$ of cases with pulmonary infection. Even in cases in which the total blood leukocyte count is normal, mild $(>5 \%)$ eosinophilia has been documented in $27 \%$ of patients by retrospective review. ${ }^{5}$ Coccidioidomycosis with pleural effusion occur in $15 \%$ of patients with pneumonia. In one small series, 12 cases of EPE were evaluated, and the degree of eosinophils in the pleural fluid ranged from $1-44 \%$ of the nucleated cells. ${ }^{6}$ Other endemic mycoses have also been associated with EPE but not as frequently as with coccidioidomycosis.

Eliciting a travel history in patients is important since a variety of tissue-migrating parasites have been associated with eosinophilia. For example, paragonamiasis can cause a pulmonary syndrome with hemoptysis, mimicking pulmonary tuberculosis. Paragonimus $s p$. can infect patients following ingestion of fresh water crustaceans from Asia, South America, and Africa. Eating crawfish in the southern United States 


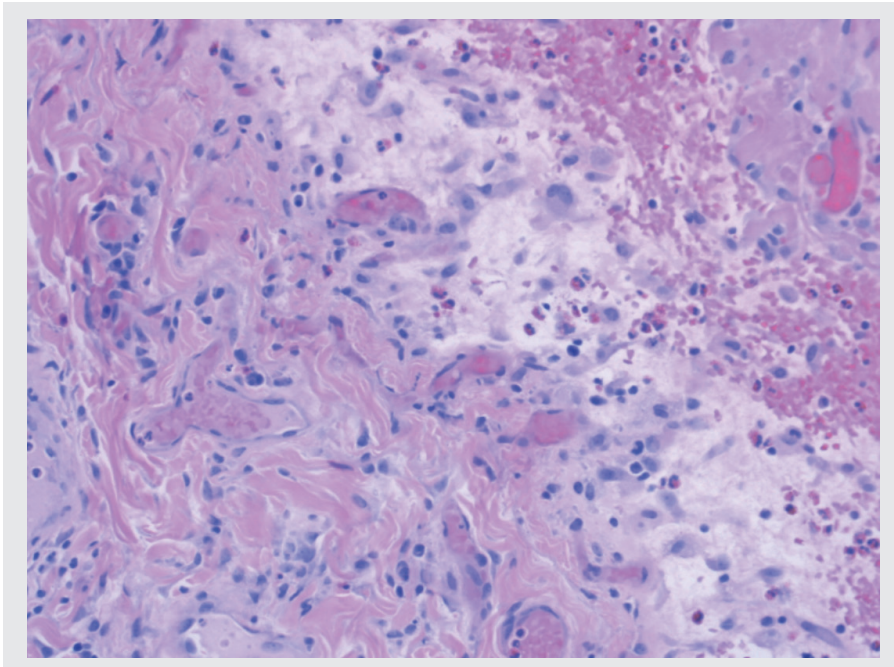

Figure 4. Pleural biopsy reveals fibrinoid eosinophilic Inflammatory infiltrates, H \& E stain.

has also been identified as a source of infection. The lung fluke often stimulates peripheral eosinophilia and EPE. Eosinophilic pleural effusion also occurs in filariasis, ascariasis, sparganosis, strongyloidiasis, and infections with other trematodes and cestodes. ${ }^{7,8}$

Evanescent eosinophilic skin lesions with EPE, as in this case, suggests the possibility of Strongyloides or a generalized auto-immune disorder. Besides larva currens, strongyloidiasis may be accompanied by peripheral eosinophilia and a form of urticaria around the buttocks and waist. These lesions last 1-2 days and recur. ${ }^{9}$ The patient presented above had no travel outside the United States. There were no pulmonary, dermal histology, or serologic findings to attribute this case to strongyloidiasis. Biopsy of skin lesions related to strongyloidiasis typically shows filariform fragments, and the enzyme immunoassay for detection of the helminth exceeds $85 \%$ sensitivity. ${ }^{10,11}$

The eosinophilic dermatoses, like EPE, may occur with or without blood eosinophilia. Most cases are related to allergic conditions, such as eczema and atopic dermatitis. Besides parasitic infestations, arthropod bites, vasculitic disorders, and blistering skin conditions such as bullous pemphigoid may exhibit similar histopathology. Eosinophilic dermatosis evolves by poorly understood mechanisms. ${ }^{12}$ Coccidioidomycosis can be associated with EPE and skin lesions such as erythema nodosum and diffuse evanescent maculopapular rashes. This patient had no pulmonary disease and no evidence of fungus on microbiologic, pathologic, or serologic analysis.

Chest trauma, pneumothorax, thoracotomy, splenectomy, and pulmonary embolism have been associated with EPE. In most of these conditions, eosinophils represent only a minority of the nucleated cells present

Table 2. Etiology of eosinophilic pleural effusions

\begin{tabular}{|l|c|l|}
\hline Cause & Relative Frequency* (\%) & Comment \\
\hline Malignancy & +++ & Half are from lung cancer \\
\hline Parapneumonic Effusion & ++ & Eosinophils rarely comprise $>10 \%$ \\
\hline M. tuberculosis & + & Eosinophils comprise $<10 \%$ NBC \\
\hline Coccidioidomycosis & ++ & Lymphocytes predominate \\
\hline Post chest surgery & + & Including Dressler's syndrome \\
\hline Transudative effusions** & + & Almost never seen with CHF \\
\hline Autoimmune disorders & - & Includes eosinophilic pneumonia \\
\hline Post chest trauma & ++ & Probably similar mechanism to surgery \\
\hline Idiopathic & ++ & Often higher \% eosinophils in fluid \\
\hline
\end{tabular}

$*_{+}++,++,+,-$correlates with $>25 \%,>10 \%,>5 \%$, or $<5 \%$ of cases, respectively

$* *$ Due to CHF, cirrhosis, nephrotic syndrome. 
in the pleural fluid. Trauma and stimulation of pleural mesothelial cells may release cytokines which recruit eosinophils to the affected zone, perhaps in some individuals due to HLA variations and other pre-determined immunologic phenotypes. Rheumatoid arthritis, ChurgStrauss, and Crohn's disease have rarely been associated with EPE. ${ }^{2,13}$

Uncommonly EPE has been reported with warfarin, propylthiouracil, sulfasalazine, and nitrofurantoin. Rarely simvastatin, isotretinoin, fluoxetine, valproate, infliximab, and crack cocaine have been implicated in EPE. ${ }^{14}$ There was no recent history of exposure to these drugs in our patient, but a remote drug use history was not obtained. Asbestosis has a notorious association with pleural disease. In a small retrospective evaluation of men exposed to asbestos with pleural disease, 7 of 10 with pleural effusions had "large numbers" of eosinophils. Pathologically, thickened chronic pleuritis was observed with fibrosis, and, in one subject, asbestos bodies were seen. ${ }^{15}$ In our patient there was no known exposure to asbestos. When asbestos exposure has caused disease, the interval between asbestos exposure to presentation is usually several decades. In this instance, there was no history of exposure nor was the pleural histopathology suggestive of this disease.

In larger retrospective reviews of EPE, nearly $20 \%$ of patients had no discernable cause. ${ }^{2}$ Evan after extensive investigations, the etiologic diagnosis for EPE may be difficult. In this case, despite a fairly extensive evaluation of the etiology in this patient, the cause of her EPE was not determined. Empiric therapy with hydroxyurea and colchicine allowed for de-escalation of high dose prednisone. Hydroxyurea has been used successfully in the management of a variety of hypereosinophilic syndromes with less short term morbidity than corticosteroids. There are case reports documenting successful treatment of eosinophilic skin disorders with colchicine. ${ }^{16}$

Since EPE is not a common syndrome no widescale therapeutic information is available. Thus, anecdotal reports comprise the major reference for our therapeutic experience of EPE.
Article citation: Lacy M. Eosinophilic pleural effusion: a case and a review. The Southwest Respiratory and Critical Care Chronicles 2020;8(33):40-46

From: Department of Internal Medicine, Texas Tech University Health Sciences Center, Lubbock, Texas

Submitted: 9/26/2019

Accepted: $12 / 27 / 2019$

Reviewer: Manish Patel MD

Conflicts of interest: none

This work is licensed under a Creative Commons

Attribution-ShareAlike 4.0 International License.

\section{REFERENCES}

1. Kalomenidis I, Light R. Pathogenesis of the eosinophilic pleural effusions. Curr Opin Pulm Medicine 2004;10:289-293.

2. Krenke R, Hasilowski J, Korczynski P, et al. Incidence and aetiology of eosinophilic pleural effusion. Eur Respir J 2009; 34:1111-1117.

3. Light R. Update on tuberculous effusion. Respirology 2010; 15:451-458.

4. Kamel A, Chabbou A, El Gharbi B. Apport diagnostiquede l'eosinophil leurale. J Rev Pneumol Clin 1989; 45:118-122.

5. Galgiani, J. Coccidioidomycosis. West J Med 1993;159: 153-171.

6. Merchant M, Romero A, Libke R, et al. Pleural effusion in hospitalized patients with coccidioidomycosis. Resp Medicine 2008:102:537-540.

7. Wang J, Luo W, Shen P, et al. Retrospective study of pleural parasitic infestations: a practical diagnostic approach. BMC Inf Dis 2019;19(576): $1-7$.

8. Emad A. Exudative eosinophilic pleural effusion due to Strongyloides stercoralis in a diabetic man. South Med J 1999;92:58-60.

9. Brooker S, Bundy D. Soil transmitted Helminths in: Manson's Tropical Disease, Farrar J (ed) 2014. Elsevier Sanders; 766-794.

10. Polenakovik H, Polenakovic S, Weinstein J. New Onset purpura in a patient with recently diagnosed temporal arteririts. Clin Inf Dis 2003;37:299-310.

11. Anderson N, Klein D, Dornink A, et al. Comparison of three immunoassays for detection of antibodies to Strongyloides stercoralis. Clin Vaccine Immunol 2014;21:732.

12. Long H, Zhang G, Wang L. Eosinophilic skin disease: a comprehensive review. Clin Rev Allergy Immunol 2016 Apr; 50(2):189-213. 
13. Boyer D, Varga S, Slattery D, et al. Churg-Strauss syndrome in children: a clinical and pathologic review. Pediatrics 2006; 118:e914.

14. UpToDate. Drug-induced pleural fluid eosinophilia. www.uptodate.com/contents, image/print?imageKey= PULM\%2F72026. Accessed 9/25/19.
15. Mattson S. Monosymptomatic exudative pleurisy in persons exposed to asbestos dust. Scan J Respir Dis. 1975;56: 262.

16. Puzas I, Alvarez M, Menendez F, et al. Wells' syndrome successfully treated with colchicine. Case Rep Dermatol 2017; 9:65-69. 